\title{
A product data dependencies network to support conflict resolution in design processes
}

\author{
M. Z. Ouertani ${ }^{\#}$, K. Grebicił **, L. Gzara-Yesilbas ${ }^{\#}$, E. Blancoł, D. Rieu** \\ ${ }^{\#}$ CRAN - UHP Nancy I, BP 239, Vandoeuvre-lès-Nancy, 54506, France; \\ E-mail: mohamed-zied.ouertani@cran.uhp-nancy.fr \\ † GILCO-INPGrenoble, 46 Avenue Félix Viallet, 38031, Grenoble Cedex, France; \\ ** LSR-SIGMA- IMAG, 2 Place Doyen Gosse-38031-Grenoble Cedex, France.
}

\begin{abstract}
It is characteristic of collaborative engineering design that precedence relationships among design activities contain information flow conflicts. Indeed, due to multiactors interaction, conflicts can emerge from disagreements between designers about proposed designs. Therefore, a critical element of collaborative design would be conflict resolution. This paper presents a methodology to identify the conflict resolution team and to evaluate the impact of a selected solution on the product data as well as on the process organisation. The approach is based on product data dependencies network. This network is composed of the handled data and the dependency links between them; two qualification dependency measures are introduced to manage these dependencies. In order to identify this network, a traceability model to track the design process is proposed. Finally, a protocol to manage the conflict resolution negotiation is discussed.
\end{abstract}

Keywords: Conflict Management, Process Traceability, Data Dependencies Network, Data Maturity

\section{INTRODUCTION}

The product design is involved in complicated interaction among multi-disciplinary design teams, exchanging parts that could be on preliminary or final state, in a distributed, heterogeneous and dynamic environment, including communication, cooperation, coordination and negotiation.

Due to multi-actors interaction, conflicts can emerge from disagreements between designers about proposed designs. In fact, each actor has his own point of view, concerns and objectives regarding the design project. Thus, a critical element of collaborative design would be the conflict management. In a collaborative design context, conflicts occur when at least two incompatible design commitments are made, or when a design party has a negative critique of another design party's actions [K1, 95]. The conflict management process could be perceived as the succession of five phases:

- Conflict detection: to consider means of detecting conflict occurrence depending on the method used to represent design constraints, design goals, design intents and design dependencies,

- Conflict resolution team identification and formation: to identify and form the team of actors (human or software) required to participate in the resolution of the identified conflict. This phase is considered as a prerequisite to conflict resolution,

- Negotiation management: to conduct and control a collaborative and competitive negotiation session, which often needs the presence of a mediating impartial senior authority or 'chair',

- Solution generation: to apply actors own domain knowledge to provide an 'optimal' solution to the considered conflict,

- Solution impact assessment: to propagate the selected solution onto the product and the design process.

In this paper, the main objective is to come up with methodological elements to allow the conflict resolution team identification and the selected solution impact on product and process organisation.

The identification of negotiation team constitutes a prerequisite for conflict resolution. Indeed, conflict resolution cannot be achieved by one single actor; it requires the gathering of different expertise areas. So to avoid much iteration in the conflict resolution process, it is highly recommended to do it in a collaborative way that seeks the interference of many actors to reach quickly a consensus. In order to provide a solution to the detected conflict, design actors have to collaborate and negotiate forming this way the negotiation team. The negotiation team participates to the conflict resolution process generating a solution to the conflict problem. The selected solution leads often to modifications on a subset of the product to design and/or on the design process organisation. Indeed, the negotiation phase leads to a solution which often implies the modification of one or more input data of the activity where the conflict has emerged, and thus, generating a cascade of modifications on the already produced data. In fact, the design process as a succession of activities which transform input data into output data, defines a precedence link between these data (i.e. an output of an activity becomes the input of successive activities).

Regarding to the impact of the selected solution on process organisation, for a process still in the pipeline, the modifications, generated by the resolution process, require an adjustment to the preliminary project organisation. However, processes that have already been 
launched and executed are re-examined and their reexecution is necessary. The organisation of the activities to be re-executed depends on the availability of the allocated resources, the delivery deadlines and the dependencies relationships between considered activities.

Therefore, negotiators identification and impact propagation are highly dependent on handled data during design process, it supposes knowing the dependency relationships between the conflict source data and the data previously produced. Thus, it arises that identifying negotiation team as well as propagating selected solution impact mainly consists of identifying the dependency relationships between technical data handled during the design process. Indeed, in collaborative design, the dependencies of engineering problems determine how and with whom designers should coordinate. Consequently, solving these two phases of the conflict management process would mean:

- Identifying the dependencies network of the data handled during the design process execution in order to define the negotiation team.

- Qualifying the data dependency links in order to propagate the impact of the selected solution.

The remaining part of the paper is organised as follows. In section 2, the data dependencies network components are described. This network is composed of nodes and arcs representing respectively the handled data during the design process and the dependency links existing between them. In section 3, the approach to identify this network is presented. It is based on a traceability model to track the design process execution. A set of SQL queries are then applied to extract the data dependencies network. The Section 4 concludes with some perspectives.

\section{DATA DEPENDENCIES NETWORK}

The data dependency network is an oriented graph composed of nodes, which correspond to the product data handled during the design process, and arcs, which correspond to the dependency relationships between these data. The rest of this section presents these network components (nodes in $\S$ II.B. and arcs in $\S$ II.C.). Before that, a case study is given to illustrate the concept of data dependencies through some design examples (§ II.A.).

\section{A. Data dependencies examples}

The case study concerns the design process of a Flexible Production System (FPS) within AIPL ${ }^{1}$ organisation (Atelier Inter-établissements de Productique - Lorraine). The system is mainly composed of an item loading station, two workstations, an item unloading station and a transportation system of items between stations. The latter is composed of a conveyor and a palette. We are

\footnotetext{
${ }^{1}$ http://www.aipl.uhp-nancy.fr

particularly interested in the workstation design process which is composed of four concurrent phases:

- Workstation frame design phase;

- Workstation energy block (pneumatic and electric energy) design phase;

- Operative part (items positioning for the product assembly) design phase;

- Automata design phase.

A special focus is given to the design of the operative part which is composed of a handler mechanism and a positionner mechanism. The handler is an arm controlled by the automata that allows moving items from the workstation stores to the palette. The positionner is made of three stores from which the handler picks up the items to assemble and of a director to guarantee the quality of the product.

The operative part design phase is split into two parallel planned activities: the handler and the positionner planned activities. At the beginning of the operative part design phase, the concerned actors have to respect the following requirements:

- the palette shape and the positioning perimeter of the wholes where to place the items;

- the automata's, operative part's and energy block's positions on the workstation frame;

- the jacks available for the actors (handler and positionner designers) to design their respective systems: three big jacks, four medium jacks, three small jacks and three rotary jacks.

According to these specifications, the handler designer defines the mechanism by using the four medium jacks available. This solution only allows reaching four possible positions; two positions on the workstation and two positions on the palette. Thus, the handler designer defined the structure, kinematics and volume of the subsystem to be designed (handler mechanism). Consequently, the positionner designer is able to define only two stores composed of a vertical stockpile with a spring each. A director is affected to each one of the stores. Likewise, the positionner designer has defined the structure and the volume of the sub system to be designed.

These characteristic data of the operative part sub systems are then used by the energy block and the workstation frame designers to be able to fulfil their respective design activities. Indeed, in order for the energy block designer to define the characteristics of his sub system, it is necessary to know the handler kinematics and the number of jacks used as well as the positionner structure and volume. As for the workstation frame designer, he will need the handler volume and the handler kinematics data as well as the positionner volume to be able to specify the frame structure and the type of material to be used. Fig. 1. recapitulates the precedence dependencies between 
handled data - an arrow defines the direction of a dependency.

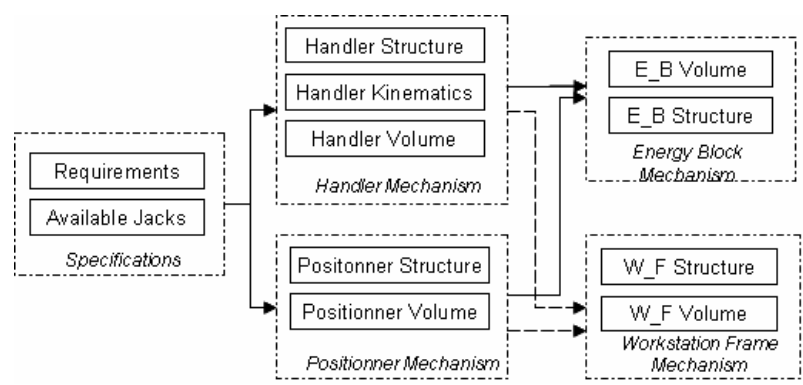

Fig. 1. Precedence dependency links between handled data

\section{B. Network nodes: product data}

Design work returns with a succession of tasks to define a new product through the use and the generation of various product data. The handled data can be of several types: structural, functional and geometrical, etc. They correspond to the various descriptions of the product, elaborated by designers during the development process, in terms of geometrical entities, functions, bills of materials, CAD models, calculation notes, simulations, etc.

Depending on the margin left to the designer to elaborate data, on the values of data properties and on the context in which it is committed, product data can evolve through different states. Grebici et al. [GBR, 05] identify four data states: draft, exhibit, enable and deliverable, according to the workspace where they are handled: private, proximity, project and public workspaces. These concepts of data state and workspace are presented in the following.

First, the designer produces a draft based on the customer requirements and on his own knowledge and competences. This piece of data is kept in the personal space of the actor. For instance, in the case study presented in $\$ 2.1$, the handler designer produced a handler mechanism draft composed of kinematics and pneumatic schemes. This first area is the private workspace; it's in that workspace that the designers produce and represent the design solution they imagine. Therefore, draft is a piece of data that one has to apply the modalities of creation and validation of hypothesis or solutions to a project or to a design problem. They are defined by a design actor individually.

Then the designer needs to confront his ideas with other actor's point of view in order to validate his design part. This corresponds to a collaborative activity requiring the contribution of several design actors who shares the collective knowledge. Thus, the designer can expose himself to critics and judgment of others. For instance, the handler designer ( $\S$ II.A.) asks the machining expert for his opinion on the possibilities to manufacture holes (support of the pieces) of a given diameter D in a manner that they are aligned on the palette. The opinion of the machining expert allows then the handler designer to define the kinematics schema. The role of this collaboration is the communication of emergent problem, the construction of a robust and convincing discourse to argue the solution, etc. According to Lecaille [Le, 03], the term exhibit is used to characterize the data handled in such exchange. This second level of diffusion is called the proximity workspace. Consequently, exhibit is a piece of data that one applies a persuasion modality in accordance with what is represented in either for convincing about the existence of a problem or for showing a solution and allowing a common construction and the point of view exchanged.

When the designers know that they are able to argue about the solution or when the delays are over, they publish their data in the integrated team common database. The data come into an official validation process and are accessible by the different actors of the project. The designer has to answer to other disciplines requests and argues about it. This level of diffusion is used when the piece of data is considered to be valid; it can be transmitted outside the personal networks. One means by validated data: those one that fulfil their users' requirements concerning the conditions about the product. Thus the design actor spreads the data to the concerned user(s) in the official space (the common database or PLM system) called project workspace. Data involved in such exchange are called Enabled. For instance, the handler designer publishes his design parts when he is ensured that the handler solution is validated. Indeed, the latter spreads the handler design parts in the official space to the positionner designer in order to allow him finishing his activity: define positionner mechanism. Hence, enabled traces are data the designer accepts to diffuse to others, after his consent or his agreement with a collective prescription to which he takes part. It is nonofficially validated objects but sufficiently convincing to be published.

Finally the design solution is validated by the hierarchy: project team manager, department manager. Thus, the already produced data are published as deliverables in the Product Data Management system (PDM system). It is the last step of the dissemination in the public area of the project. Therefore, deliverable are data that transmit a strong regulation. They have been formally verified and validated (by hierarchy). They are those contractual supports to being communicated to the customer.

\section{Network arcs: dependency links}

Among the criteria that characterise links between elements (objects, tasks, design team, etc.), the dependency link criterion remains the more studied in research works and the less simple one to treat in the collaborative design modelling. Many definitions of dependency link are proposed in literature. For Kusiak and Wang [KW, 95], a dependency between variables is 
the effect of change in a value of one variable on another variable, whereas for Jin and Wang [JW, 00], two components are said to have dependency relation if any of the two can not be completed without the other. These definitions reveal that two kinds of dependencies may exist between two product data: dependency at creation and dependency at modification. Two data are said "dependent at creation" if the creation of one of them depends on the creation of the second one - this corresponds to the Jin and Wang definition. Two data are said "dependent at modification" if the change of one of them implies the modification of the second one - this corresponds to the Kusiak and Wang definition.

The rest of this sub-section is devoted to characterise these dependencies. The main focus is on quantifying and qualifying the dependency link through the concepts of dependency degree and dependency nature.

\section{1) Dependency degree}

Depending on the dependency context (at creation or at modification), there exist different attributes that can be used to express the dependency degree between two elements in design.

Dependency at creation measure: Some research works have attempted to define attributes to express the link between data at creation, such as: the relevance, the usage and the completeness of the upstream data to the downstream data [CDHM, 05] [WBO, 97]. We are particularly interested in the completeness attributes which draw the actual data variation interval. The actor will express how should be the variation interval of the consumed data. Higher is the completeness attribute value, smaller would be the input data interval variation. Even if the completeness of information is defined by its user with regard to his needs to produce another data ${ }^{2}$, it represents an absolute measure of the "at creation" dependency compared to the relevance and usage. In this paper, we are concerned with the estimation of the completeness attribute as the "at creation dependency" measure.

TABLE I

CONSTRUCTED ATTRIBUTE FOR COMPLETENESS

\begin{tabular}{cl}
\hline Attribute level & \multicolumn{1}{c}{ Description of the attribute level } \\
\hline 0 & $\begin{array}{l}\text { Weak: the input data could be given below a certain } \\
\text { maximum value }\end{array}$ \\
& $\begin{array}{l}\text { Not Vital: the input data should be given within a certain } \\
\text { value range }\end{array}$ \\
2 & $\begin{array}{l}\text { Vital: the input data should be given with the smallest } \\
\text { value range } \\
\text { Extremely Vital: } \text { the input data should be precisely } \\
\text { given }\end{array}$ \\
\end{tabular}

2 A data could be considered as complete in order to be used for a given data and not for another one.
The completeness of data provided by an activity is arbitrarily categorized in four levels according to a discrete and subjective measurement scale using structured expert interviews. Table I summarises the completeness attribute values.

Dependency at change measure: among the attributes proposed in science management and engineering design works to define the dependency, the following are the most relevant to measure the dependency "at change": Level Number [S, 81], Importance Ratings [PE, 94] and Probability of Repetition [BE, 02]. We are particularly concerned with the estimation of the last measure of dependency since it constitutes the hardest to obtain input for simulating a development process that involves iteration. The probability of Repetition reflects the probability of one element (activity, data) causing rework in another. Krishnan et al. define the dependency measure as the multiplication of both attributes: Variability and Sensitivity [KEW, 97].

- Variability ${ }^{3}$ is the likelihood that the output data provided by one task would change after being initially released [YFC, 99]. Since the variability is associated with the stability of a particular element, each output element has its own variability value. The variability concerns the results of an upstream activity output that constitutes input data for the downstream activity. It is difficult, if not impossible, to come up with a universal objective measurement scale for information variability to be used in all product development situations. Therefore, a discrete, subjective measurement scale for this measure is constructed using the techniques for constructing subjective attributes as described in [Ke, 92]. The estimated variability of information provided by a task is arbitrarily categorized in four levels, each having a numerical value, as shown in Table II.

TABLE II

LEVELS OF DATA VARIABILITY - ADAPTED FROM [YFC, 99]

\begin{tabular}{cl}
\hline Attribute level & \multicolumn{1}{c}{ Description of the attribute level } \\
\hline 0 & Not variable: the output data don't vary \\
1 & Low Variability: the output data varies but few \\
2 & Moderate Variability: the output data is instable \\
3 & High variability: the output data is very instable \\
\hline
\end{tabular}

- Sensitivity is the degree to which work is changed as the result of absorbing transferred information (or data). In another words, this attribute expresses the sensitivity of output data (performed during a downstream activity) if the input data variation occurs (in the upstream activity producing this input data). Sensitivity depends on the level of dependency between two particular elements. Table III describes the three subjective levels of an

\footnotetext{
${ }^{3}$ Defined as the speed with which complete information is created for transmission in Krishnan et al 1997
} 
element sensitivity developed using the techniques for constructing subjective attributes as described in [Ke, 92].

TABLE III

LEVELS OF DATA SENSITIVITY - ADAPTED FROM [YFC, 99]

\begin{tabular}{cl}
\hline Attribute level & \multicolumn{1}{c}{ Description of the attribute level } \\
\hline 0 & $\begin{array}{l}\text { Not sensitive: output data sensitivity is null to most } \\
\text { input data changes } \\
1\end{array}$ \\
$\begin{array}{l}\text { Minor sensitivity: output data sensitivity is low to most } \\
\text { input data changes } \\
\text { Moderate Sensitivity: output data sensitivity is medium } \\
\text { to most input data changes } \\
\text { Major sensitivity: output data sensitivity is high to most } \\
\text { input data changes }\end{array}$ \\
\hline
\end{tabular}

In order to quantify the dependency link between two data, the dependency at creation and dependency at change measures are aggregated to one criterion to express the dependency degree between two data.

Therefore, the three attributes; completeness, variability and sensitivity are aggregated to measure the dependency degree (cf. Fig. 2.). As they are complementary attributes, a multiplicative utility function is utilised in the aggregation of the variability and sensitivity attributes $\left(\mathrm{V}^{*} \mathrm{~S}\right)$. Furthermore, more the completeness is high (from partially used until totally used) more the required rework is long. Thus, for a given variability and sensitivity values, more the completeness is important, more the iterations are long and more the dependency degree is high. In the case when the variability value is " 0 " and the completeness value is different from " 0 ", the dependency degree value must be different of " 0 ", since that a not null completeness implies a dependency at creation. Then, the dependency degree formula is:

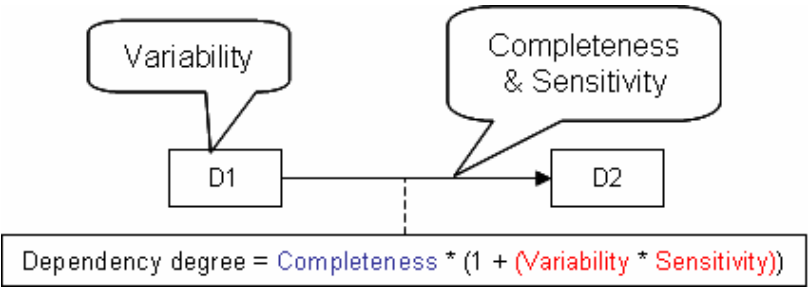

Fig. 2. Dependency degree between data D1 and D2

Accordingly, the resultant range value of the dependency degree is an integer between 0 and 30 , whereas $\{0,1,2,3$ and 4$\}$ denotes a weak dependency and a low risk of rework, and $\{15,20,21$ and 30$\}$ denotes a very high dependency and a high risk of rework. The values $\{5,6$, 7 , and 8$\}$ and $\{9,10,12$ and 14$\}$ describe respectively a moderate and a high dependency and risk of rework.

\section{2) Dependency nature}

For a better management of the conflict resolution process, it is interesting to distinguish the nature of the dependency link that exists between the data. This would lead to the elaboration of various network filters providing the project manager with different points of view of the handled conflict source. In this network, various types of dependencies can be distinguished respectively from a resource perspective, a process perspective and a product perspective.

- Inter-domain: dependency between data produced by actors form different domains: For example, the data jack and workstation frame are inter-domain dependent since they are produced by actors from the pneumatic domain for the handler designer and the mechanical domain for the workstation frame designer.

- Intra-domain: dependency between data produced by two actors from the same domain: dependency between data produced by two actors from the same domain. For example, the data jack of the handler mechanism and the data store of the positionner mechanism are intra-domain dependent since they are produced by actors from the same domain; the pneumatic domain.

- Inter-phase: dependency between data produced in two different phases. These data are message flow supported since they are exchanged through shared space or e-mails between activities from different phases. For example, the data handler and the data palette are inter-phase dependent since they result from two concurrent phases: the handler and the palette design activities.

- Intra-phase: dependency between data produced within the same phase. These data are sequence flow supported since they are exchanged through sequence links between activities of the same phase. For example, the data jack and the data sensor are intra phase dependent since they result from the same activity; i.e. the handler design phase.

- Inter-Bill of Material (BoM): dependency between elements from different BoMs such as Functional or Structural etc. of the same product or two different products. For example, the linked data handler kinematics and energy block structure are inter-BoM dependent since they are attached respectively to the functional and the structural BoMs.

- Intra-Bill of Material: dependency between the same BoM, of the same product or of two different products. For example, the linked data handler volume and positionner volume are intra-BoM dependent sine they are attached to the geometrical BoM.

\section{APPROACH TO BUILD UP THE DATA DEPENDENCIES NETWORK}

The objective of the proposed approach is to come up with methodological elements that allow the identification of data dependencies and then their qualification. In order to do so, the first step is to trace the real design process execution by storing it in a database system. Then, SQL queries are applied on the obtained data to extract data dependencies network. The rest of the section proceeds as follows. Paragraph III.A, develops the design process constructs to be traced. Paragraph III.B, presents the resulting traceability model which allows storing the 
process execution data in a database system. Finally, the use of the database to obtain the data dependencies network is illustrated in $\S$ III.C.

\section{A. Traceability Constructs}

Traceability in product development is defined by Hamilton and Beeby as the ability to discover the design history of every feature of a product [HB, 91] Traceability dimensions can be described by answering the basic questions adopted from Zachman [Z, 87]:

Where are the traceable items being handled, which activity did consume/produce the items?

What are the traceable items - design objects, requirements, design decisions etc.?

Who are the resources playing different roles in the creation, modification and use of those items?

Why - How are the items being created, modified or evolved?

When are items being created, modified or evolved?

Each of these questions is developed below.

\section{1) Where?}

In order to answer the managerial imperatives due to the costs, delays and quality constraints, the design process is seen as a decomposed project in a different design phases. A design phase is closed following a decisional activity (decision deadline) making it possible either to stop the design process, or to continue it (on going to the following process). Each design phase is organised according to an execution planning of the preset activities. This modelling level corresponds to the organisation of the planned design activities (based on intermediary results) in order to fit with the realisation needs of the project planning and of the resources management. This organisation relates to identified information but not yet formally validated. Such project management allows organising the conception phase as well as to integrate the management constraints (costs, quality and delay) in the design activities realisation.

However, a description of the design phase in term of preset and planned activities is not sufficient. Indeed, the emergent and innovative design properties make the design activities being not completely predictable. A first global decomposition of the process can be defined at early stage. The fine decomposition of a design process is determined according to the ongoing design, to the produced product data and to the evolution of the context in which the design is carried out.

Thus, two management levels of the process exist, the prescribed one (the coordination according to the costs, quality and delay imperatives) and the emerging one (according to the creation needs of the design solution and environment). The emergent management level corresponds to the creative and non-preset organization.
Indeed, the design activities are non-planned and can be known only a posteriori.

Consequently, a design process is modelled as a succession of phases where each phase is decomposed on planned activities. Each planned activity can be in its turn decomposed into a set of non-planned activities. A class "Design action" is also defined to factorise the properties and the common links to the three classes "Phase", "planned Activity" and "non-planned Activity".

\section{2) What?}

During collaborative design process execution, phases, planned activities and non-planned activities are dataflow supported. In deed, an activity consumes input data to produce output data, and communicate them to other activities in order to achieve the design objectives. When the consumer activity as well as the producer activity belongs to the same phase or planned activity, the communication proceeds accordingly to three succession types; AND, OR and XOR. The exchanged data are thus said to be sequence flow supported. In the case the data exchange is between two activities of different phases, the exchanged data are said to be message flow supported.

As discussed in section II.B, a data could take four different states; draft, exhibit, enabled and deliverable according to its evolution during the design process execution. In this work, only enable and deliverable state are tracked; in order to assure the system consistency and to ensure the fact that exchanging preliminary data, does not induce additional iterations in the design process execution. Indeed, the data with the Draft and Exhibit state are not enough mature and are subject to multiple iterations as well as their possible delete during the design process.

In term of data modelling, we define a class "Technical Data" related to the class "Design Action" according to two associations: one to indicate the input and the other to indicate the output. Input data could be of three types; design environment constraints (specifications, managerial decision, etc.), sequence flow supported data (produced by an upstream design action in the same phase or in the same planned activity) or message flow supported data (produced by an upstream design action in a different phase or planned activity). In addition, and in order to be able to measure the dependency degree in the network of dependences to be built, the concepts of variability, sensitivity and completeness (cf. § II.C.1) are associated to the input or output data.

\section{3) Who, Why, When and How?}

During the design process execution, design actions are carried out by resources (Who?) which could be human or material resources. They act in different roles in order to achieve a given objective (Why?) during a time 
represented by the design action beginning and end date (When?). Actors deploy their know-how and competencies in order to make decision and propose solution that they must justify later. The justification (How?) is an explanation of why an artefact, or some part of an artefact, is designed the way it is [LL, 91]. The key issues for the justification concept herein adopted are articulated as questions, with each issue followed by one solution that responds to the issue. Arguments support the adopted solution and could be of Requirement-based, Function-based, Rule-based, Case-based and/or standard.

\section{B. Traceability Model}

To allow storing the various records tracing the design process execution in a database system, the various constructs discussed above are formalized in the following UML model (Fig. 3.).

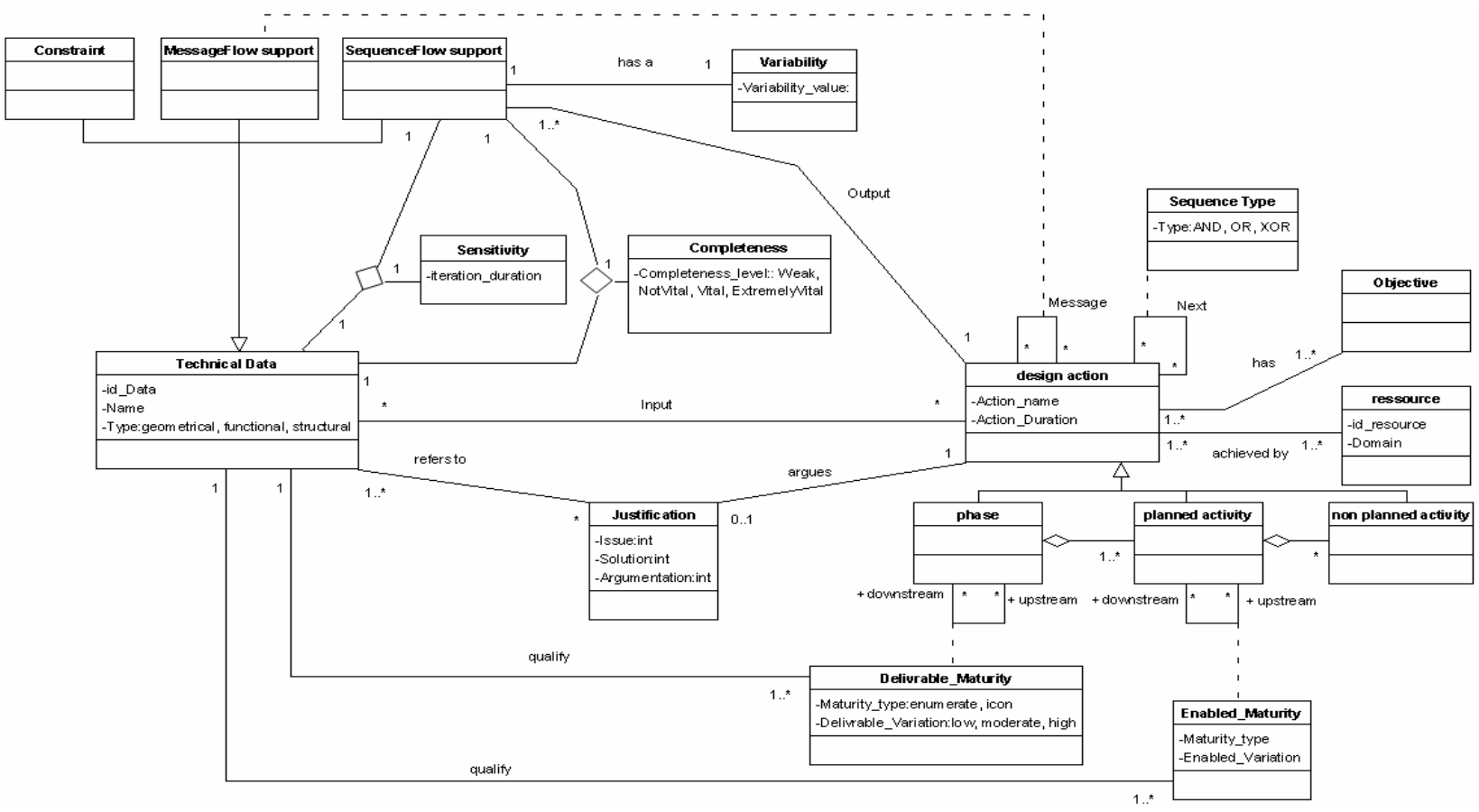

Fig. 3. UML model to achieve design process traceability

\section{From traceability model to Data Dependencies Network}

Based on the model presented in Fig. 3., a traceability tool can be defined. This tool is composed of an $a$ posteriori workflow engine which allows declaring the design ongoing as a workflow. This workflow engine allows each actor involved in the design process to declare, when achieving his design action:

- the phase, planned activity or non planned activity he is executing (name, Id...),

- the objective if his design action,

- the input and the output data he used and generated with the associated sensitivity, variability and completeness measures, as well as the maturity attributes (for enabled or deliverable maturities),

- the justification of the choices he made during the design action,

These declarations must be done by all the involved actors during each one of their design actions. Once the design workflow is declared, the captured constructs are stored in a database whose tables correspond to the various classes of the traceability model. Indeed, each workflow element declared by actors corresponds to an instantiation of one of the database tables. Then, a set of
SQL queries can be applied to the stored data in order to collect the dependencies of the conflict source data. These queries are applied as many times as it is necessary in order to identify the whole conflict dependant data. Once the dependency network is extracted, it is possible to identify the negotiation team and the design activities to be re-executed. Fig. 4. illustrates the described approach to obtain the data dependency network from the traceability model.

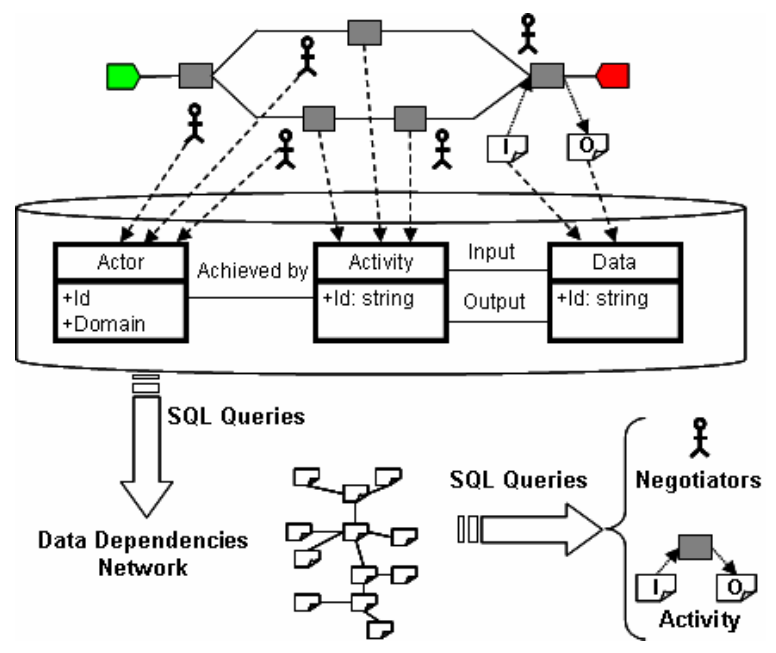


Fig. 4. Data dependencies network identification approach

Once the data dependencies network is identified, the data on which depends the piece of data source of conflict are identified through the network backward coverage; the piece of data source of conflict is the starting point. Then, SQL queries are applied in order to identify the actors responsible of theses data realisation. The formers (i.e. these actors) will then be part of the negotiation team that will resolve the conflict.

When a solution to the conflict is selected, the impacted data are identified through the data dependency network (i.e. a forward cover of the network starting from the piece of data to which leads the selected solution).

\section{CONCLUSIONS}

In this paper, a methodology has been introduced to support conflict management; in particular negotiation team formation and impact propagation on product data and process organisation. The proposed methodology is based on a process traceability method support to building up the data dependencies network composed of nodes i.e. technical data and arcs i.e. dependency links. A tool is under development in order to implement this methodology within a PLM solution; a first validation will be done within the FPS workstation design example.

However, further thoughts remain to be carried out for the process re-organisation problematic. In fact, the proposed methodology presents a support for conflict management. Based on data dependencies network, designers are able to identify negotiators and to propagate modifications on previously defined product data. These modifications often require a re-execution of activities producing data to be modified. Actors can ensure different roles in several concurrent projects whose delivery dates are predefined. Consequently, the processes reorganisation proves to be a difficult task since it depends on availabilities of the actors able to execute these modifications.

In order to do so, it is necessary, first to identify the projects to reorganise, since a data can be used in several concurrent processes; and next, to define the needs to these processes and the objectives to achieve with them. The third phase would be to model and to analyse these processes. A description of the different aspects of the processes (i.e. technological, resources, etc.) is then to be given. The execution and the coordination of the activities, the exchanged data and the allocated resources are to be analysed. Finally, based on the result of the modelling and the analysis phases, the process can be properly redesigned. Indeed, strategies to manage the overlapping of coupled product development activities are to be proposed. These strategies are based on the dependency degree attributes (§ II.C.1). In fact, the combination of the three attributes: completeness, variability and sensitivity will allow answering questions such as: when should downstream activity act on upstream data? How should the activities be overlapped when the downstream activity cannot work on preliminary data?

While as for the planned processes but not yet executed, metrics are to be proposed in order to guarantee an optimised adjustment for the impact assessment. These metrics constitute a dashboard to the project manager and concerns essentially the number of impacted activities, the impacted phases, the resources to be assigned (competences, availabilities, etc.).

\section{REFERENCES}

[BE, 02] Browning T. R., Eppinger S. D., Modelling Impacts of Process Architecture on Cost and Schedule Risk in Product Development IEEE Transactions on Engineering Management, vol. 49, no. 4, pp. 428-442, 2002 .

[CDHM, 05] Culley S.J., Davies S., Hicks B.J. and McMahon C.A., An assessment of quality measures for engineering information sources, 15th International Conference on Engineering Design, August 15-18, 2005 .

[GBD, 05] Grebici K., Blanco E., Rieu D.. Toward Non Mature Information Management in Collaborative Design Processes, 15th International Conference on Engineering Design, August 15-18, 2005.

[HB, 91] Hamilton, V-L., and Beeby, M-L., 1991, Issues of Traceability in Integrating Tools, Proc. IEE Colloquium Tools and Techniques for Maintaining Traceability During Design

[JW, 00] Jin, Y., and Wang, K., 2000 Modelling and managing dependencies in engineering design, in CIRP Annals, Vol.49 (2).

[Ke, 92] Keeney,R., Value Focused Thinking (Cambridge, MA: Harvard University Press), 1992.

[KEW, 97] Krishnan V., Eppinger S.D, Whitney D.E, A Model-Based Framework to Overlap Product Development Activities, Management Science Vol.43, N4 PP 437--451, 1997

[K1, 95] Klein, M., 1995, Conflict management as part of an integrated exception handling approach, Artificial Intelligence for Engineering Design, Analysis, and Manufacturing, vol. 9, 259-267.

[KW, 95] Kusiak, A., and Wang, J., 1995, Dependency Analysis in Constraint Negotiation, IEEE Transactions on Systems, Man, and Cybernetics, Vol. 25, No. 9, 1301-1313.

[Le 03] Lecaille P., 2003, "La Trace Habilitée, une Ethnographie des Espaces de Conception dans un Bureau d'Etudes Mécanique: 1'Echange et l'Equipement des Objets Grapho-numériques entre Outils et Acteurs de la conception", thèse de doctorat INPGrenoble, novembre 2003.

[LL, 91] Lee, J., Lai, K. What's in design rationale. Human-Computer. Interaction, 6 (3-4), 251-280 1991.

[PE, 94] Pimmler T. U., Eppinger S. D.. Integration Analysis of Product Decompositions, ASME Conference on Design Theory and Methodology, Minneapolis, MN, pp. 343-351, 1994.

[S, 81] Steward, D., System Analysis and Management: Structure, Strategy and Design, (New York: Petrocelli Books), 1981. 
[WBO, 97] Wilkilson, G.L., Bennett, L.T and Oliver, K.M. Evaluating the quality of internet information sources, Educational Technology (3), $52-59,1997$.

[YFC, 99] Yassine A., Falkenburg D. and Chelst K., "Engineering Design Management: an Information Structure Approach", International Journal of Production Research, Vol. 37, no. 13, 1999.

[Z, 87] Zachman, J-A., 1987, A Framework for Information Systems Architecture, IBM Systems Journal, Vol. 26, No. 3, 276-292. 